\title{
Aerodynamics of bird flight
}

\author{
Rudolf Dvořák ${ }^{1, a}$ \\ ${ }^{1}$ Institute of Thermomechanics v.v.i.,Academy of Sciences of the Czech Republic, 18200 Prague 8, The Czech Republic
}

\begin{abstract}
Unlike airplanes birds must have either flapping or oscillating wings (the hummingbird). Only such wings can produce both lift and thrust - two sine qua non attributes of flying. The bird wings have several possibilities how to obtain the same functions as airplane wings. All are realized by the system of flight feathers. Birds have also the capabilities of adjusting the shape of the wing according to what the immediate flight situation demands, as well as of responding almost immediately to conditions the flow environment dictates, such as wind gusts, object avoidance, target tracking, etc. In bird aerodynamics also the tail plays an important role. To fly, wings impart downward momentum to the surrounding air and obtain lift by reaction. How this is achieved under various flight situations (cruise flight, hovering, landing, etc.), and what the role is of the wing-generated vortices in producing lift and thrust is discussed.The issue of studying bird flight experimentally from in vivo or in vitro experiments is also briefly discussed.
\end{abstract}

\section{Introduction}

People have always been fascinated by the perfection of the bird flight, and in the past centuries many enthusiasts attempted to copy it, unfortunately, in most cases with no success. About the end of the fifteenth century Leonardo da Vinci systematically studied the flapping bird wings and observed and described very carefully the bird's flight.

The first successfully man-operated airborne vehicle by Otto Lilienthal (about the end of the nineteenth century) had fixed wings. However, Lilienthal also studied in detail the bird wing structure and discovered the importance of cambered profiled wings. Horatio F.Phillips about the same time had investigated the aerodynamics of curved double-surfaced airfoils, and in 1884 had it even patented. All this knowledge was applied by Wright brothers, who in 1904 performed a first succcessful flight with a heavier-than-air-airplane of their own design. Their airplane had also fixed wings, and, fixed wings had all other man designed and operated airplanes in the whole century which followed. It was a magnificent century of hectic research and development of new aircrafts, and at its end people proudly and immodestly claimed that in flight abilities their airplanes had reached almost a bird-like perfection.

It is quite interesting that during the twentieth century people scarcely ever sought inspiration in the animal world. Rather, they used the most sophisticated instrumentation, available mainly towards the end of the century, to realize that what they have achieved, birds have possessed for millions of years.
However, towards the end of the last century a new interest has appeared in small autonomous or remotely controlled flying vehicles. The so called micro-airvehicles (MAV) were expected to be used in aerial reconnaisance in open as well as confined spaces, in monitoring polluted areas which people cannot enter, and (last but not least!) in many military applications. Soon it has become clear that if these vehicles should also be able to carry a reasonable payload, they would have to have either rotating or moving wings. Since the MAVs operate at about the same Reynolds numbers as birds, bats and larger insects, it has recently opened a new interrest in aerodynamics and flight mechanics of all of them. In this paper, however, we will deal only with avian flight, i.e., with the flight of birds.

\section{Bird wings}

To fly, birds must have wings. Unlike airplanes they must have moving wings, i.e., they must have either flapping or, in one exceptional case (the hummingbird), even oscillating wings. Only such wings can produce both lift and thrust - two sine qua non attributes of flying.

First airplane designers, like Otto Lilienthal and Horatio Phillips, believed that it is enough to copy the bird wing shape and the wing profile. Unfortunately, the bird wing is not a simple curved compact surface like any of the vintage airplane wings.

The basic structure of a bird's wing resembles the human hand, only proportions of the bones are different, as much as they are different in every bird species. The hand section of the wing provides the main dynamic

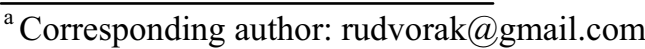


control for the bird. It represents about $80 \%$ of the wing length in small birds, who have to manoeuvre in constraint environment. In larger birds, using the wings mainly for soaring, gliding or slow flapping, the hand section is much smaller, and the wing is ruled by the arm bones extending from 40 to $60 \%$ of the wing span (Fig.1), [1], [2], [3].

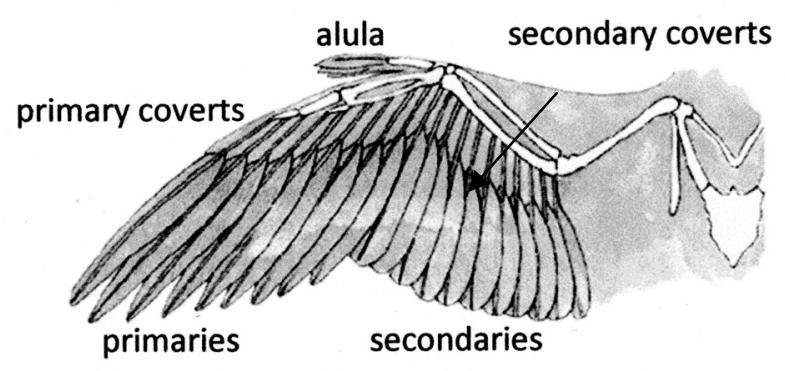

Figure 1. Flight feathers.

Primaries are connected to the bird's fingers. Primaries are the longest and narrowest of the outer feathers (the remiges), and they can be individually rotated. These feathers are the main source of thrust, mostly generated on the downstroke of flapping flight. On the upstroke the primaries are separated and rotated, reducing air resistance while still helping to provide some thrust. Remiges on the wingtips of large soaring birds like condors or vultures also allow for spreading the feathers, reducing thus the creation of wingtip vortices.

Secondaries are connected to the ulna. They remain close together in flight (they cannot be individually separated like the primaries) and help to provide lift by creating the airfoil shape of the bird's wing. Secondaries are usually shorter and broader than primaries.

The alula feathers are not flight feathers in the strict sense; however, they are very useful in slow flight. Attached to the bird's "thumb", they lie normally flush against the leading edge of the wing and detach only at higher angles of attack creating a gap between the alula and the rest of the wing (compare with slats on airplane wings). Birds can thus avoid stalling at low speeds or at landing.

The actual shape of the bird wing is made of two organized sets of feathers - in the first set are the flight feathers anchored in the digits (primaries) and the ulna (secondaries). There are also three sets of the so called coverts which act as a protective cover for all or part of the folded primaries and secondaries.

The vanes of each feather have hooklets that lock the feathers together, giving thus the wing - if locked - the necessary strength to withstand the lift force and to maintain its shape. Each feather has a bigger side and a lesser side. Moreover, the shaft bends slightly off its longitudinal axis with the lesser side to the front and the bigger side to the rear of the feather (Fig.2). The feather anatomy enables rotation of the feather in its follicle. During the up-stroke the bigger side is pressed down, opening thus the wing like a jalousie and allowing air to slip through the wing. This considerably reduces the upward resistance of the wing during upstroke (see again
Fig.1). The integrity of the wing is reestablished during the downstroke, when the pressure acts from below and the feathers lock again to form a compact wing surface.

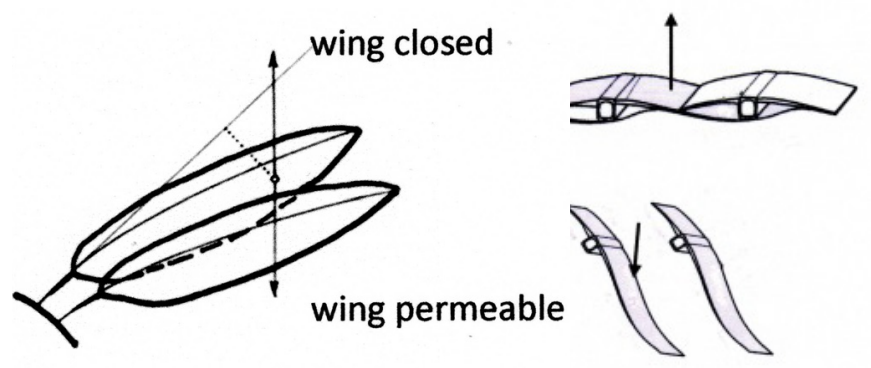

Figure 2. Forces on the flight feathers during downstroke (a) and upstroke (b).

The large flight feathers at the wing tip (primaries, outer remiges) contribute greatly to the production of lift. These feathers form the tip slots which can considerably reduce the induced drag by acting as winglets, i.e., by making wings effectively non-planar and eliminating thus the intensity of the tip vortex by spreading the vorticity vertically.

The flight feathers together with the covert feathers are responsible for morphing the wing shape. The many degrees of freedom of the wrist and elbow bones and all these feathers make the bird wing highly flexible in changing the wing chord and span, and in the spanwise twisting and bending of the wing, thus helping to maintain attached flow and reduce induced drag at the wing tip.

\section{The bird flight}

The command of flight is essential for the day-to-day struggle for survival, foraging, escaping from enemies, as well as for managing so demanding flight situations like landing and taking off. All this can be achieved only if the wings have the possibility of changing the lift in fractions of a second. These abilities are supported by a very short reaction time, which is almost by one order lower than in humans. It is important that birds have the capabilities of adjusting the shape of the wing (incl. camber and the wing area) according to what the immediate flight situation demands, as well as of responding almost immediately to conditions the flow environment dictates, such as wind gusts, object avoidance, target tracking, etc

There are two kinds of moving wings operating in two different modes -the flapping mode (birds, bats) and the oscillating mode (insects). Both are characterized by a complex unsteady and threedimensional motion of the wings, changing in every instant with the new position of the wings, [1],[2],[4],[5]. The wingbeat cycle in birds has two kinematically distinct phases - the downstroke phase (the wing goes usually down) and the upstroke phase (the wing returns to its initial position). The whole cycle highly depends on the purpose of the flight and has therefore various modifications even in the same species 
The downstroke is often called the power stroke, as it provides the majority of lift and thrust, while the upstroke is a recovery stroke.

The motion of the wings is quite complex and depends on the aim of the flight or on the situation.

Close to the body, there is very little up and down movement of the wing, while movement of the outer part may be quite vigorous. The outer part of the wing has to twist and even change the profile so that each part of the wing can maintain the optimal angle of attack. During the downstroke, the angle of attack is increased and is decreased during the upstroke. As the wing twists, and as the outer part of the wing moves downward, the aerodynamic force is directed forward and the wing produces thrust. During the upstroke, the outer part of the wing must return with as minimum drag as possible, usually with zero angle of attack. The bird partially folds its wings, reducing thus the wingspan. As a rule, the primaries open, allowing passage of air through them, reducing further the drag. The inner part of the wing behaves in a different way. There is little up-and-down movement there so that this part of the wing continues to provide lift and functions more or less as a fixed wing. Thus lift does not fall down to zero between the downstroke phases of the wingbeat cycle.

Birds can very efficiently change the wing area. This is typical namely of seabirds who can almost instantly switch from the cruising flight with fully extended wings to a dive with wing close to the body. Even when descending for landing, the area of the wing is decreased by raising the inner part of the wing upwards (this at the same time increases the bird's stability). By changing the wing area, birds can lower the aerodynamic load on the wing and thus decrease the risk of flow separation.

The primary feathers at the wing-tip of birds that soar over land spread out to form typical slotted tips (see, e.g., Fig.6). It has been shown by theoretical calculations as well as by wind tunnel measurements that these slotted tips can reduce the induced drag by acting as winglets on airplane wings, i.e.,by spreading vorticity both horizontally and vertically [1],[6].

The reduction of drag on wings with elliptical lift distribution as compared with planar wings without slotted tips amounts up to $25 \%$.

Slotted wing tips are typical of birds that soar over land. Birds that soar over the sea (like gulls and albatrosses) usually have longer and narrower wings with pointed tips to reduce the induced drag. Slotted tips enable shorter wings than the narrow pointed wings without tip slots. Short wings are lighter than the narrow wings with pointed tips, producing the same amount of lift because they have lower root bending moment. They are also more manoeuvrable - they can roll and yaw at higher angular velocities for a given tip speed. All that is advantageous for birds flying in constraint environment like forests and soaring in unpredictably changing gusts over land. Sea birds are adapted to different living conditions. They operate at higher average speeds, and slotted tips may become a liability at these speeds, increasing profile drag more than reducing induced drag.
What are the means the bird wing has to increase instantly the lift or suddenly stop the flight at an perching point?

An airplane wing has a variety of flaps, slots and slats which effectively alter the shape of the wing, increase its curvature and allowing thus to divert more air, and thus create more lift [1],[5]. However, this alteration also increases drag, which helps a landing airplane to slow down, but necessitates more thrust during take-off. Slats perform the same function as flaps, changing temporarily the wing shape to increase lift on takeoff and landing. They deploy from the wing leading edge, like the flaps deploying from the trailing edge. Besides these, airplane wings have wing brakes to slow down the plane at landing. To minimize the induced drag at the wing tips modern airplanes have winglets to reduce the aircraft's drag by partial recovery of the tip vortex energy..

The bird wings have several possibilities how to obtain the same functions as the above described arrangements on airplane wings. All are realized by the system of flight feathers (see Fig.1), together with the ability to increase the camber, and also to exploit the aerodynamics function of the tail [7].

The tail is an integral part of the bird's lifting system. Unlike the tail of an aircraft, it is very close to the trailing edge of the wing and helps to make the wing morphology more versatile. It has a variable area and a variable aspect ratio. It maintains the stability and balance, especially in slow flight, where the tail is widely spread to enhance the flow over the wing in a similar way as an extended slotted flap from the trailing edge of an aircraft wing.

The underwing coverts (clearly seen in Fig.3) open automatically along the leading edge at high angles of attack. They operate as an automatic high-lift device, analogous to a Krueger flap, increasing the wing's effective camber [8].

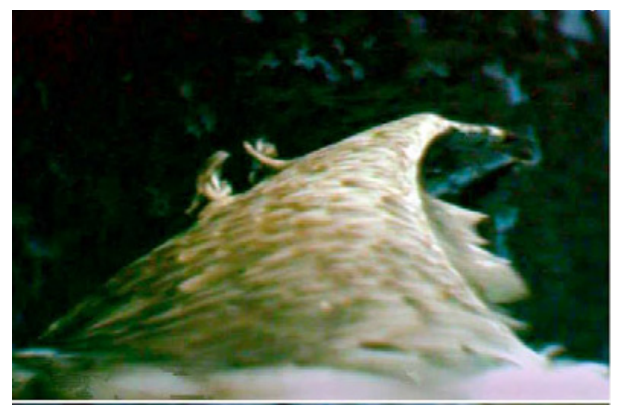

Figure 3. Deployed underwing coverts.

Take-off is one of the most energetically demanding manoeuvres of flight Small birds facilitate the take-off by jumping up, while larger birds take a run-up to receive the take-off velocity or simply try to receive it by facing into the wind. When perching on a branch or cliff all they need to start is to drop off into the air.

Landing is also a problem for many large birds with high airspeeds. In order to lose height and velocity rapidly prior to landing, large birds often use a series of sideslips.

The actual landing manoeuvre of all birds proceeds in a very similar way. 
During the landing manoeuvre the tail is widely spread, extending thus the wing surface and its camber to generate maximum lift as possible. Close before perching, the tail moves up pushing the bird upwards (or even partly backwards) to the position where the wings stall and the bird slowly perches down. To enhance braking immediately before perching, the bird may use a few wingbeats with wings fully extended and set against the flow direction, the tail again lowered down to increase the lift (see, e.g., Fig. 4).

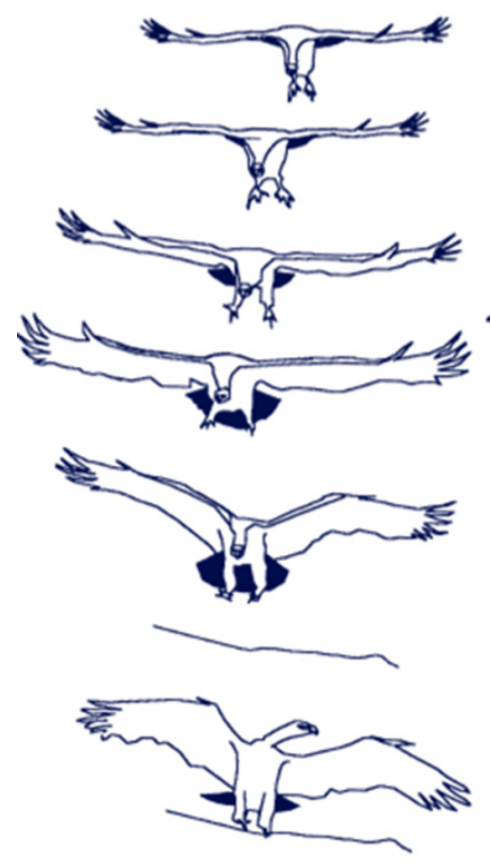

Figure 4. This picture is adapted from the ALR Thomas (1993) paper [7], and shows the last sequences of the landing manoeuvre of a white-backed vulture (Gyps africanus).Time between successive images is $1 / 5$ of a second. Note especially the movements of the tail.

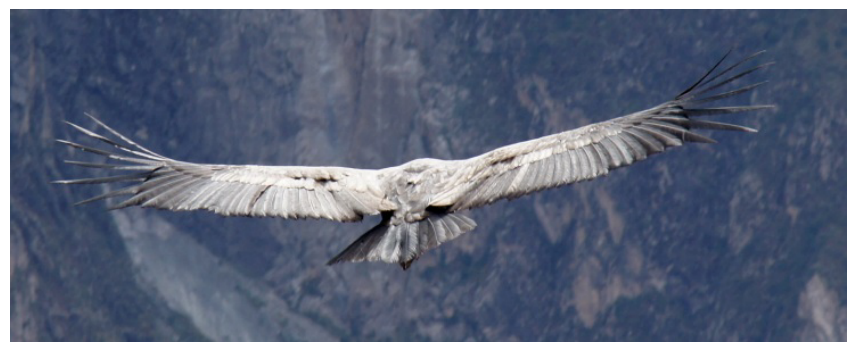

Figure 5. Picture of an Andean condor (Vultur gryphus) preparing for landing. Notice the raising up of the covert feathers on about one third of the wing span from the body and the widely spread tail.

Under normal flight conditions the tail acts independently of the wings as a kind of a control surface (both horizontal and vertical). Measurements in the wake have not detected the presence of separate wake of the bird's body. This means that from the aerodynamics point of view the tail has to be treated as an integral part of the whole bird, at least together with the wings.

Flight at low speeds or flight with high angles of attack when high lift is required is inevitably accompanied by a risk of flow separation. Separation starts near the trailing edge but very quickly moves upstream to the low pressure peaks at the wing crest or near to the leading edge, leading ultimately to a complete stall. However, this reverse flow can be slowed down or even eliminated by self-activated flaps - spanwise fences on the wing upper surface [1] formed by covert feathers raising up automatically when necessary (see Fig.5).

The function of a leading edge slot in bird wings takes up the alula - feathers anchored in the first digit (see Fig.6).

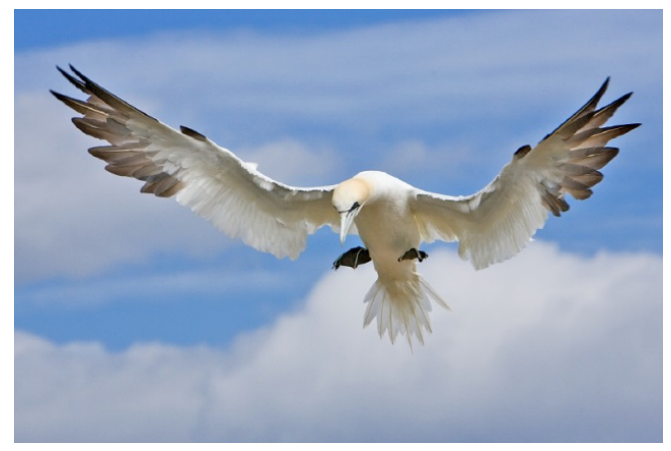

Figure 6. Landing manoeuvre of the Northern gannet (Morus bassanus) documents well the use of the alula, the tail and wing morphing.

The wing-tip-slots do not eliminate completely the vortices at the wing tips (induced vortices), especially in lower aspect-ratio-wings, as, e.g., in passerines. They are generated during the downstroke phase and strengten by gradually engulfing the trailing edge vortex sheet. The strong downward-induced velocity thus effectively enhances the lift. Traditionally, tip vortices are considered as a nuisance increasing the drag, however, in low-aspect-ratio-wings they can favourably enhance the lift. The downward velocity behind the flyer is accompanied by an upward velocity field outside the wake, and in V-flight formation of migrating birds this can be used by the follower's wing as an energy saving contribution to the generated lift (Fig.7) [1].

The increased downwash is also beneficial at near-toground flight (ground effect, skimming over the water surface), giving the bird considerable performance advantages, including a reduction in mechanical flight power of as much as 35\%, compared with values for flight out of ground effect.

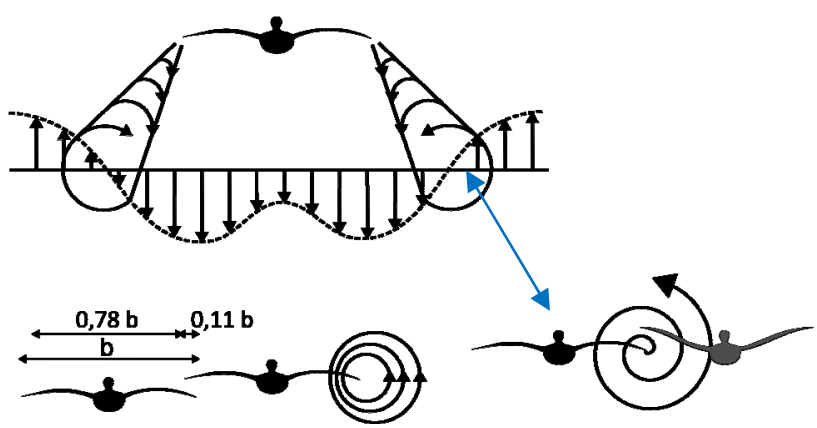

Figure 7. Flight in V-formation to save energy. 


\section{How do birds actually generate lift?}

To fly, wings impart downward momentum to the surrounding air and obtain lift by reaction. This is Newton's 3rd Law, which says that action and reaction are equal and opposite to each other. The lift force, which counterbalances the weight, is obtained from Newton's 2nd Law.

To obtain the downward momentum, we have to know the downward component of the velocity deflected by the wing and the mass flow which is proportional to the air density, the wing area and the downward component of the velocity. This velocity component depends on the flight speed and on the angle of attack.The mass flow due to the deflected wing depends on the wing area, wing curvature, and in birds also on various lift-increasing-manipulations with the feathers. However, in principe, lift depends primarily on the angle of attack!

With the only exception of a hummingbird, birds generate lift and thrust by flapping the wings. This is a complex unsteady and threedimensional motion of the wings, changing at every instant with the new position of the wings. The aerodynamic analysis of bird flight was usually based on the quasi-steady assumption, according to which all instantaneous forces on a flapping wing in unsteady motion are assumed to be those corresponding to steady motion at the same instantaneous velocity and attitude. This assumption may be misleading (as is definitely the case when dealing with insect flight) and it is recommended to consider even the aerodynamics of the bird flight as unsteady.

Of course, wings not only generate lift and thrust, they cause drag as well. Optimally designed wings have to bring the air from upstream to downstream with as little loss of momentum, i.e. loss of drag, as possible and at the highest lift as possible.

Lift and drag are two components of the resulting aerodynamic force acting on the wing. The actual size of the components of the resulting aerodynamic force on vertical and horizontal axes depends primarily on the argument and magnitude of the wingbeat velocity (see, e.g., Fig.8 )

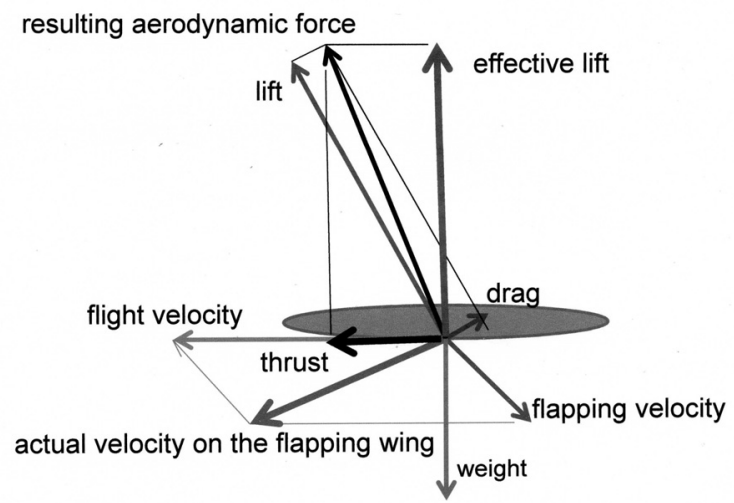

Figure 8. Aerodynamic forces on a flapping wing

The many facets of bird wing shapes and movements make a unifying theory of how lift is generated a difficult task. The only fact we can take for granted is that the mechanism of lift generation is ruled by vortices. The vortices drive in the surrounding air and impart downward momentum to this air [9]. The way how they do it depends on the wing shape, flapping velocity, as well as on the flight velocity. We will discuss here three possible models: high aspect ratio wings in cruising flight, high aspect ratio wings in more "energetic" flight and low aspect ratio wings.

The bird wings, however, are far from being twodimensional [10]. The tip vortices make the flow threedimensional and enter into nonlinear interaction with the spanwise shedding vortices. This interaction is, of course, significantly influenced by the wing shape, wing aspect ratio and the angle of attack. Especially on low aspect ratio wings the tip vortices can even completely destroy their original character of the vortex streets. The above described mechanism of producing thrust is thus valid only for cruising flight of birds with large aspect ratio wings with minimized tip vortices, like, e.g., condors, vultures or albatrosses.

During the downstroke phase of high aspect ratio wings typically two counterrotating vortices are generated - on the leading edge and on the trailing edge (Fig.9) [9], both being shed with the stream Fig.10). This vortex couple drives the air downward, generating thus lift in the opposite direction. On low aspect ratio wings the tip vortices on either wings dominate, forming again a couple to generate lift. Naturally, in this case interaction with the spanwise oriented vortices cannot be avoided (see, e.g., Fig. 12), as apparent from the complex wake structure [11].

Wake is a kind of footprint which any flying body leaves behind in the surounding atmosphere. It represents not only the loss of momentum, comprising most of the losses generated by the flying body, but its structure also tells a lot about the behaviour of the body during the flight, especialy when the body contours are changing.
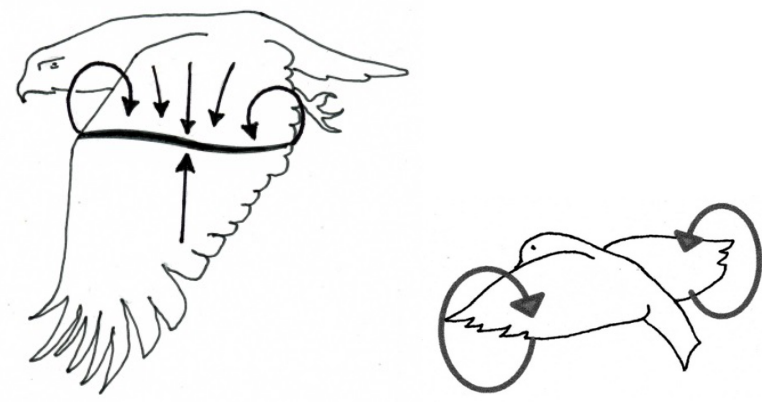

Figure 9. Vortex couples generating lift, a)high aspect ratio wings, b) low aspect ratio wings 


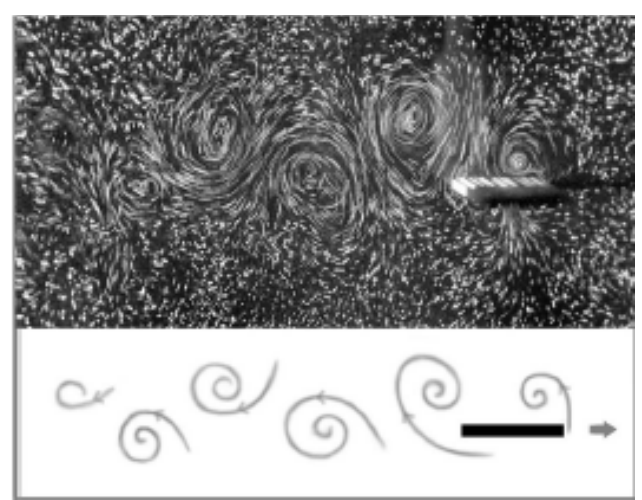

Figure 10. Vortices shed off with the stream.
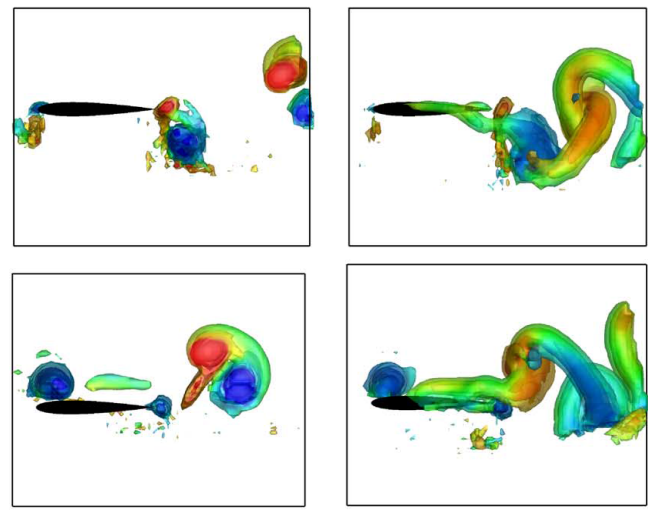

Figure 11. The left pictures show vortex shedding on a $2 \mathrm{D}$ profile at two different times, the right pictures refer to the ends of a3D profile at corresponding times and velocities and show interaction with the tip vortices.

The wakes behind fixed 2D wings with blunt trailing edges, or behind 2D blunt bodies, have a typical character of a Kármán vortex street (Fig.12a), [1].
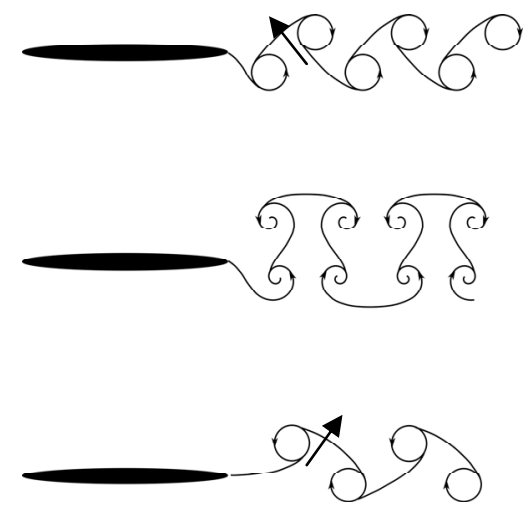

Figure 12. Vortex streets in wakes behind 2D fixed and oscillating airfoils.a) is a classical Kármán vortex street producing drag, b) mild lateral oscillations of the airfoil, the vortices are changing, c) higher frequency of oscillations generate a vortex street with opposite sence of vortex rotation to a), producing thus thrust.

However, provided the wing is heaving (moving in a direction perpendicular to the velocity of the oncoming flow), the vortex pattern in the wake may become different, being now dependent on the frequency of this movement. Behind a slowly heaving wing the wake is still similar to that behind the blunt trailing edge of a fixed wing. The sense of rotation of the vortices results from the momentum deficit behind the wing and represents net drag. By increasing the frequency the vortices in the wake gradually acquire opposite sense of rotation, the wake becomes "inverted" and the wing produces thrust (see, e.g., Fig.12c). This effect has been known since Knoller (1909) and Betz (1912) perceived that flapping a wing in a free stream flow resulted in an effective angle of attack with a normal force vector containing both lift and thrust components. This effect was experimentally demonstrated later by Katzmayr (1922) and revisited by Lai and Platzer in several papers published about the end of the twentieth century (see, e.g.,[12]. The effect of inverted Kármán vortex street is used by slowly soaring birds for occasional increase in lift. They slowly flap the wings up and down and generate thus the necessary lift.

Wakes behind flapping wings are much more complex than wakes behind fixed wings. Some time ago it was generally accepted that the downstroke is important in generating lift and thrust, and, therefore, it is sufficient to study only wakes in the downstroke phase. However, it has soon been realized that both the downstroke- and upstroke-generated wakes are equally important in building up the wake character. Moreover, it has also been proved that wake depends not only on the species in question, but in the same species it depends also on the particular gait (purpose of flight).

The wake develops while the wing flaps down and up and especially during downstroke the induced vortices play a considerable role. The tertials (the feathers nearest to the body) act in most cases as fixed wings and provide weight support throughout the whole cycle. Analysis of direct pressure measurements has suggested that the wingtip may be active even during the upstroke, providing some thrust. Fig.13 is just an example of a real wake to demonstrate its complexity [13].

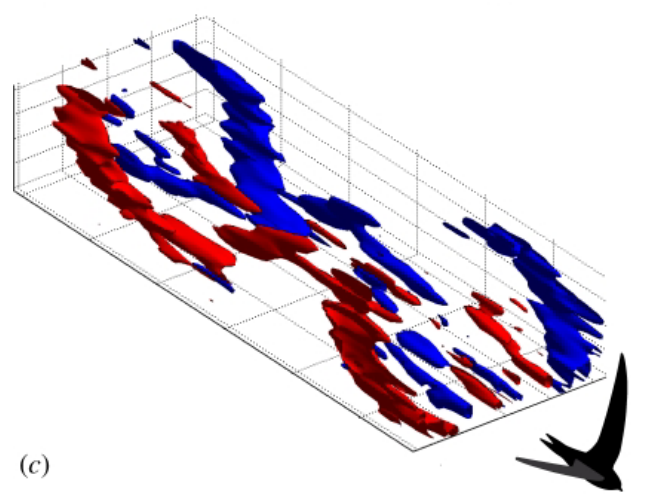

Figure 13. Iso-surfaces showing 1.5 wingbeats for the speed $7.7 \mathrm{~m} \mathrm{~s}^{-1}$. Iso-value is $60 \mathrm{~s}^{-1}$. The view is oriented so as if the bird was flying obliquely towards the observer from left to right. Red-coloured patches represent positive circulation (clockwise rotation as seen in direction of flight) and bluecoloured patches represent negative circulation (counterclockwise rotation as seen in direction of flight).Adapted from [13].

\section{Experimental bird aerodynamics}

This is a conference on experimental fluid dynamics so it is quite natural to ask whether it is possible to learn more about bird's flight from laboratory or in vivo experiments. 
Cruising flight has been filmed many times and these films, however interesting they may be, do not answer the most interesting (from the aerodynamics point of view) sequences of bird's flight like take-off and landing. This has changed in the last decades thanks to high speed cinematography and recently even to videorecording using miniaturized cameras placed directly on the bird's body (see, e.g., [8], or Fig.3 of this paper).

The wind tunnel tests were aimed at two groups of problems - direct measurement and evaluation of lift and drag from measuring in wakes, and in vivo measurements of the origin of these forces.

Wakes behind flying birds can be investigated experimentally in wind tunnels by application of several highly sophisticated experimental methods developed for

experiments on airplane models.

It would be hardly possible to rely on any pressure measurements in the wake behind flapping wings. The only chance has been offered by the digital PIV (see, e.g.[14], or Fig.14 and Fig.15). However, a qualified interpretation of these results was first possible only after a CFD simulation of the same situation was available.

Experiments [14] were carried out using a closedloop, low-turbulence wind tunnel designed for bird flight experiments. Four juvenile thrush nightingales Luscinia luscinia L. were caught, and after a period of acclimatization they were exposed to daily flight training for more than two months prior to experiments, under conditions that gradually resembled the experiment. Training started with low ambient light conditions, introduction and maintenance of fog particles, and occasional bursts of high intensity laser light.

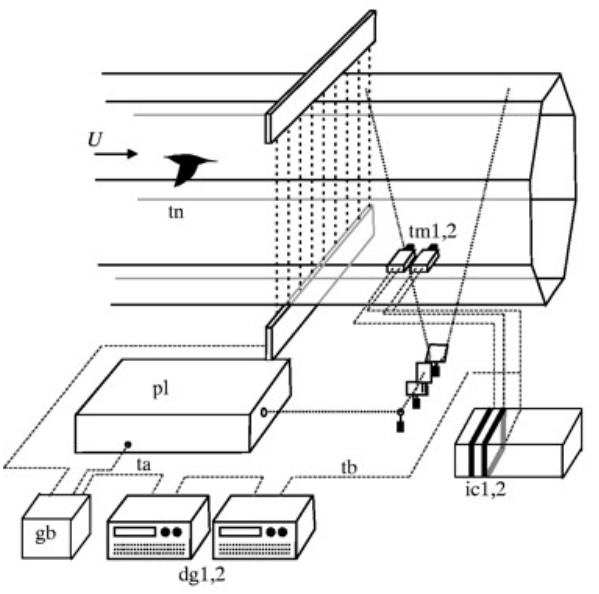

Figure 14. The bird (tn) is flying at constant speed, $U$ is the independently controlled speed of the wind tunnel. Two delay generators $(\mathrm{dg} 1,2)$, configured to run off a single crystal base timing clock, generate synchronised timing pulses to control the dual-head Nd:YAG (pl) laser and the asynchronous reset (tb) for the two CCD array cameras (tm1,2). Digital images are acquired at independent interface cards (ic1,2) and transferred directly to PC RAM. The laser timing pulses are gated (gb) with the summed output from an array of LED-photodiode pairs so that if any one or more beams are interrupted by the bird, laser output stops (Modified from[14]).

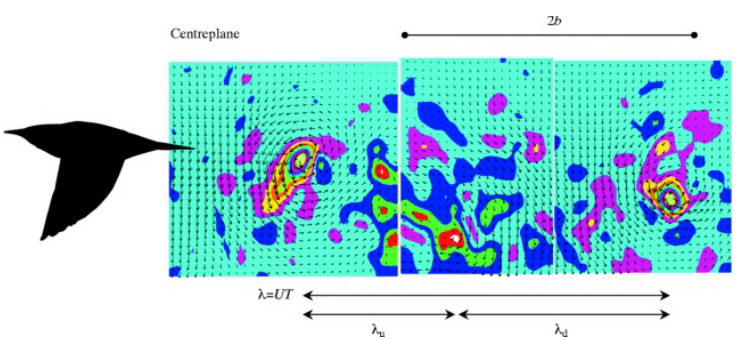

Figure 15. A reconstruction from three consecutive frames to show the vortex wake over slightly more than one wingbeat cycle. The wake is shown as if left in still air by the bird passing from right to left, from [14].

Recently, a special wind tunnel has been designed the so called Aerodynamic force platform (AFP), [15], to measure directly the forces on bird wing during the bird flight. The AFP is a box instrumented with load cells enclosing the object (the bird) that generates the unsteady fluid force. According to Newton's 3rd law applied to a fluid, the unsteady net fluid force needs to be supported by an equal and opposite net force that acts on the control volume boundary.

Whereas the unsteady and convective terms are significant in the bulk fluid, they vanish at the surface of the AFP. The contour integral is thus simplified and when even the viscous shear forces acting on the wall can typically be ignored, to obtain the net aerodynamic force we can just integrate the pressure as measured on the wall.

The aerodynamic forces on the bird wing during takeoff and landing were successfully measured in vivo by pressure transducers and accelerometers built in directly in the wing (see [16] and Figs. 16).

A
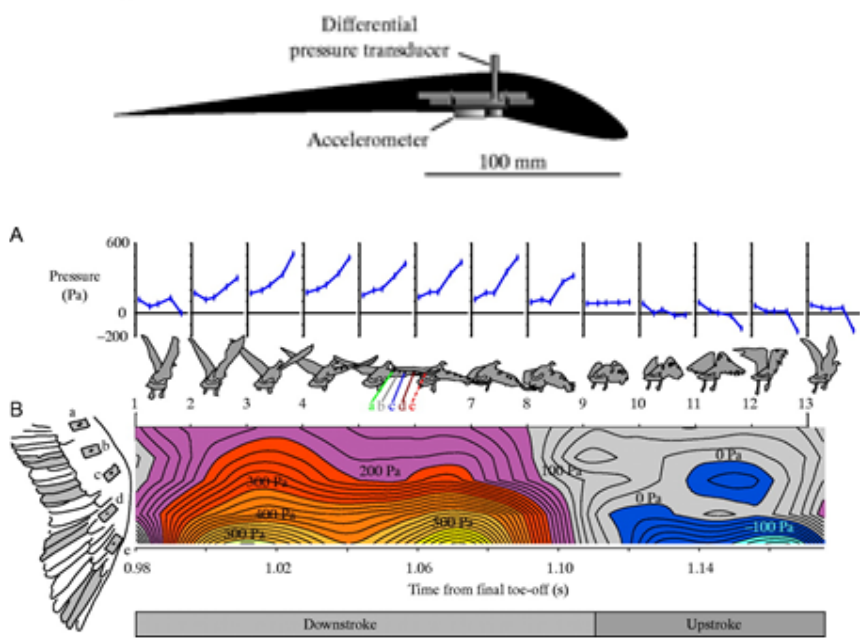

Figure 16. Pressure distribution related to inematice for a wing over a single flap during take-off flight, but five flaps after lift-off. The pressure distribution along the wing (A) show snapshots separated by $4 / 250 \mathrm{~s}$. The contour plot (B) shows the pressure distribution through time as it would be mapped to an outstretched wing. Contours are calculated assuming a linear distribution of pressure with spanwise distance between the five measurement sites indicated on the wing image (labeled a-e). Positive pressures indicate a ventral-to-dorsal sense; the wing base maintains a weight-supporting sense throughout the wingstroke cycle.(adapted from[16]). 
Also, further in vivo measurements were successfully performed with surgically implanted strain gauges of mechanical work and power output from the main muscles (see Fig.17, or[17]).

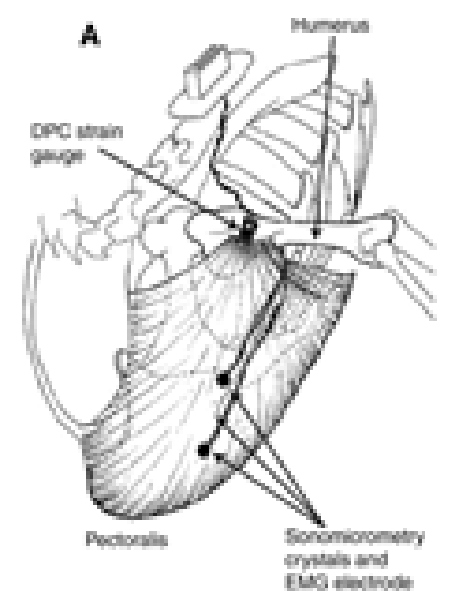

Figure 17. In vivo measurements of mechanical work and power output from the pectoralis. The primary downstroke muscle has surgically implanted strain gauges calibrated to measure force from bone strain on the deltopectoral crest of the humerus and sonimicrometry crystals to measure muscle length.

\section{Closing remarks}

Investigation into animal flight has its value not only for aircraft designers of the MAVs, but it can be inspiring for all those who do research in fluid dynamics.

The sole issue of generating lift and thrust by moving wings may even motivate us to reassess our knowledge on the same issue from the classical fixed wing aerodynamics.

\section{References}

1. Dvořák R.: How they fly(In Czech), Academia, Praha, 2015

2. Azuma A.: The Biokinetics of Flying and Swimming, Springer V., Tokyo, 1992

3. Šestakova G.S.: Strojenije kryl'jev i mechanika poljota ptic, Izd. Nauka, Moskva, 1971

4. Lighthill M.J.:Aerodynamic aspects of animal flight, In: Swimming and flying in nature, Plenum Press,pp.423-491

5. Norberg U.M.: Vertebrate Flight, Springer V., Berlin Heidelberg New York, 1990

6. Tucker V.A.: J.Exp.Biology, 180, 1993, pp.285-310

7. Thomas A.L.R. : Phil.Trans.Royal Soc., London, B, Vol.340, pp.361-380

8. Carruthers A.C., Thomas A.L.R., Taylor G.K. J.

Exp. Biology, 210, 2007, pp.4136-4149.

9. Dvořák R.,Applied and Computational Mechanics, Vol.8, No 2, 2014, pp.147-156

10. Shyy W., Y. Lian, J. Tang, D. Viieru, and H. Liu: Aerodynamics of Low Reynolds Number Flyers, Cambridge Aerospace Series, No22, 2008

11. Calderon D. E., D. J. Cleaver, I. Gursul, Z. Wang Physics of Fluids, 26, 071907 (2014)
12. Platzer M.F.,K.D.Jones,J.Young,J.C.S.Lai, AIAA Journal,46,2008,No 9,pp.2136-2149

13. Henningsson et al.,J R Soc Interface, 2011 June6, 8(59);pp.807-816

14. Spedding, G. R., Rosén, M. and Hedenström, A. J. Exp. Biology, 206, 2003, pp.2313-2344

15. Lentink D., Haselsteiner A.F., Ingersoll R. $J$ R Soc Interface, 2015, 12:20141283.

16. Usherwood J.R., T.L.Hedrick, A.A.Biewener, J.Exp.Biology, 206, 2003, pp.4051-4056

17. Tobalske B.W., J.Exp.Biology, 210, 2007, pp.31353146 